\title{
A rod model for large bending and torsion of an elastic strip with a geometrical imperfection
}

Received: 3 December 2018 / Published online: 22 March 2019

(C) The Author(s) 2019

\begin{abstract}
We consider an initially horizontal curved elastic strip, which bends and twists under the action of the varying length of the span between the clamped ends and of the gravity force. Equations of the theory of rods, linearized in the vicinity of a largely pre-deformed state, allow for semi-analytical (or sometimes closedform) solutions. A nonlinear boundary value problem determines the vertical bending of a perfect beam, while the small natural curvature additionally leads to torsion and out-of-plane deflections described by the linear equations of the incremental theory. Numerical experiments demonstrate perfect correspondence to the finite element rod model of the strip. Comparisons to the predictions of the shell model allow estimating the range of applicability of the three-dimensional theory of rods. Practically relevant conclusions follow for the case of high pre-tension of the strip.
\end{abstract}

Keywords Elastic rods · Incremental equations $\cdot$ Spatial Kirchhoff rods $\cdot$ Shell finite elements $\cdot$ Asymptotics

\section{Introduction}

Problems of finite spatial deformations of elastic rods with coupled torsion and bending are mainly treated using finite element or other variational formulations; examples of solutions based on differential equations are rare to find in the literature. This increases the value of such semi-analytic or even closed-form solutions, in particular for studies of parameter sensitivity, asymptotic treatment, etc. Particularly interesting are equationbased solutions for practically relevant or fundamental problems. Thus, it can be analytically shown that a straight rod with a symmetric cross section assumes a helical form under the action of a dead-end moment, see Eliseev [1]. An iterative algorithm featuring sequential solutions of a nonlinear boundary value problem (BVP) for the spatial bending of a clamped-free circular rod by an out-of-plane force was suggested in [2]. Huynen et al. [3] as well as Belyaev and Eliseev [4] treated constrained torsion of a rod in a spatially curved channel. For the analytic treatment of instability and supercritical torsion of annual rings with unsymmetric cross section and natural out-of-plane curvature, see Vetyukov [5]. Dias and Audoly [6] developed and applied an extended rod-like theory of folded elastic strips; Audoly and Seffen [7] considered stability and various supercritical configurations of naturally curved elastic strips and compared to experiments. Fang and Chen [8] considered small oscillations of a largely deformed rod with clamped ends by first solving the nonlinear problem of plane deformation and then using a linearized version of the theory, which is methodologically close to the present study.

Yu. Vetyukov $(\varangle) \cdot$ C. Schmidrathner

Vienna University of Technology, Getreidemarkt 9, 1060 Vienna, Austria

E-mail: yury.vetyukov@tuwien.ac.at

C. Schmidrathner

E-mail: christian.schmidrathner@tuwien.ac.at 
Equations, linearized in the vicinity of a pre-stressed but undeformed configuration, are frequently used for stability analysis. Thus, problems of lateral-torsional buckling of deep beams under various kinds of loading have become classical, see Eliseev [1], Vetyukov [2], Simitses and Hodges [9]. Buckling of pretwisted straight rods has been extensively discussed by Glavardanov and Maretic [10] and by Ziegler [11]. Michell's instability of twisted elastic rings was re-discovered by Goriely [12]. For lateral-torsional buckling of thin-walled channel-like rods of open profile at bending and compression, see [13]. While using incremental formulations, the mentioned studies feature trivial pre-stressed configurations of the rod, which are either straight or circular.

Many essentially nonlinear solutions to problems of in-plane deformations of rod structures are available in the literature. Thus, a contact of a closed elastic rod with cylindrical pulleys is analyzed in [14-16]. Denoël and Detournay [17] used Eulerian kinematic formulation in the problem of deformation of an elastica within a channel contacting both its walls. For post-buckling of shear-deformable beams, we refer to Humer [18]. Stability of non-trivial plane equilibrium configurations of flexible rods is studied in comparison with experimental results by Levyakov and Kuznetsov [19].

The present contribution combines all mentioned kinds of analysis. We decompose coupled flexuraltorsional deformation of a naturally curved rod into large in-plane bending of a perfect beam and an additional incremental out-of-plane deformation and torsion because of the curvature. The resulting configuration follows after solving two BVPs, the nonlinear one for the large pre-deformation and the linear one with the equations of the incremental theory. Further we demonstrate the asymptotic character of the solution for flat rods, whose out-of-plane bending stiffness is high, by deriving and solving the equations for the leading order terms. Closedform solution are discussed for specific combinations of parameters, which either result in a particularly simple differential equation, or at which the approximate solution is easy to obtain.

The semi-analytical results are in a perfect correspondence with the outcome of finite element simulations with classical Kirchhoff rod models; see [20-22] for the numerical schemes. We also involved a shell finite element solver [23] and compared deflections and rotations against data, obtained with the shell model of the strip. Despite good correspondence for moderately thick strips, we observed significantly different results decreasing the thickness-to-width ratio of the cross section of the rod. Attributing the discrepancies to the shell-specific deformation forms, which cannot be described by the kinematics of a rod, we thus determine the little known boundary of the range of applicability of the Kirchhoff theory to strips with a thin cross section at coupled bending and torsion. We conclude the paper by a practically relevant study of the effect of axial pre-stretch on the lateral and torsional deformations of a horizontally clamped strip owing to its natural curvature in the horizontal plane.

\section{Theory of Kirchhoff rods: nonlinear and incremental}

We will employ the equations of the nonlinear theory of rods in the form suggested by Eliseev [1,24], see also [2]. For alternative but mathematically equivalent presentations, we refer to Antman [25] as well as Simitses and Hodges [9]. Equations of the theory follow from the principle of virtual work, applied to a material line with three translational and three rotational degrees of freedom of particles. In the classical theory, conditions of inextensibility and absence of shear impose certain constraints between the rotations of particles and variations of the axis of the rod. In the following, we consider the rod to be unshearable, but extensible, thus extending the range of considered examples.

Particles of the rod are identified by their material coordinate $s$, which is the arc coordinate in the undeformed state. Position vectors of particles $\boldsymbol{r}(s)$ define the geometry of the axis, and the orientation of particles is determined by the rotation of three basis vectors $\boldsymbol{e}_{k}(s)$; see Fig. 1. Constraints of the classical theory allow choosing the third basis vector in the tangent direction, such that the unit tangent vector is

$$
e_{3}=\boldsymbol{t}=\boldsymbol{r}^{\prime} /\left|\boldsymbol{r}^{\prime}\right| \text {. }
$$

The strain measure for axial extension is traditionally introduced as

$$
\varepsilon=\left|\boldsymbol{r}^{\prime}\right|-1, \quad \boldsymbol{r}^{\prime}=(1+\varepsilon) \boldsymbol{t} .
$$

The rate of change of the local basis along the axis of the rod is determined by the vector of twist and curvature $\boldsymbol{\Omega}$ :

$$
\boldsymbol{e}_{k}^{\prime}=\boldsymbol{\Omega} \times \boldsymbol{e}_{k}, \quad \boldsymbol{\Omega}=\frac{1}{2} \boldsymbol{e}_{k} \times \boldsymbol{e}_{k}^{\prime}=\Omega_{k} \boldsymbol{e}_{k}
$$


summation over the repeating index is implied, and $\Omega_{k}$ are the components in the local basis.

In the undeformed reference state, we have the axis $\stackrel{r}{r}(s)$ and the orientations of particles $\stackrel{\bullet}{\boldsymbol{e}}(s)$, and

$$
\left|\stackrel{\boldsymbol{r}}{\prime}^{\prime}(s)\right|=1, \quad \stackrel{\circ}{\boldsymbol{\Omega}}=\stackrel{\circ}{\Omega}_{k} \stackrel{\circ}{e}_{k} .
$$

Along with $\varepsilon$, the deformation from the reference state to the actual one is described by the vector

$$
\boldsymbol{\kappa}=\left(\Omega_{k}-\stackrel{\circ}{\Omega}_{k}\right) \boldsymbol{e}_{k}=\kappa_{k} \boldsymbol{e}_{k} .
$$

The components $\kappa_{1,2}$ are responsible for bending, and $\kappa_{3}$ shall be interpreted as torsion of the rod. Computing these strain measures, we deal with the components $\Omega_{k}$ and $\Omega_{k}$, which are, respectively, written in the actual basis and in the reference one. If we write the tensor of rotation of a particle from the reference to the actual state $\mathbf{P}$, then its derivative along the axis is expressed similarly to the time derivative of the rotation tensor of a rigid body (as if $\kappa$ was angular velocity and $s$ was time):

$$
\mathbf{P}=\boldsymbol{e}_{k} \stackrel{\circ}{\boldsymbol{e}}_{k}, \quad \mathbf{P}^{\prime}=\kappa \times \mathbf{P} .
$$

The internal force factors are the force $\boldsymbol{Q}(s)$ and the moment $\boldsymbol{M}(s)$, which act from the particle $s+0$ on the neighboring one $s-0$. The equations of balance in a state of static equilibrium

$$
\begin{aligned}
& \boldsymbol{Q}^{\prime}+\boldsymbol{q}=0, \\
& \boldsymbol{M}^{\prime}+\boldsymbol{r}^{\prime} \times \boldsymbol{Q}+\boldsymbol{m}=0
\end{aligned}
$$

feature the external distributed forces $\boldsymbol{q}$ and moments $\boldsymbol{m}$, which are counted per unit material length of the rod. The constitutive relations

$$
Q_{t} \equiv \boldsymbol{Q} \cdot \boldsymbol{t}=b \varepsilon, \quad \boldsymbol{M}=\mathbf{a} \cdot \boldsymbol{\kappa}
$$

relate the internal axial (also known as normal) force and the moments to the strain measures. The physically linear model features the tension stiffness $b$ and the symmetric tensor of stiffness for bending and torsion $\mathbf{a}$, which has constant components in the local basis:

$$
\mathbf{a}=a_{i j} \boldsymbol{e}_{i} \boldsymbol{e}_{j} \Rightarrow M_{i} \equiv \boldsymbol{M} \cdot \boldsymbol{e}_{i}=a_{i k} \kappa_{k} .
$$

Conventionally, the torsion and the bending are decoupled, and

$$
\mathbf{a}=-E \boldsymbol{t} \times \mathbf{J} \times \boldsymbol{t}+a_{t} \boldsymbol{t} \boldsymbol{t}
$$

in which the bending stiffness is determined by the tensor of moments of inertia $\mathbf{J}$ of the cross section and the Young modulus $E$. The torsional stiffness $a_{t}$ follows from the classical Saint-Venant problem of pure torsion as the integral of the Prandtl stress function times the shear modulus.

No constitutive relation exists for the transverse components of the force vector $Q_{1,2}$, as the corresponding work-conjugate deformation forms are kinematically constrained in the considered model. Instead, they follow from the equilibrium conditions (7). The system of equations is completed by the boundary conditions.



Fig. 1 Rod as a material line with positions of particle $\boldsymbol{r}(s)$ and their orientations $\boldsymbol{e}_{k}(s)$ depending on the material coordinate $s$, $t=e_{3}$ 
Small increments in the external forces $\boldsymbol{q}^{\cdot}$ and moments $\boldsymbol{m}^{\cdot}$ result in small displacements $\boldsymbol{r} \cdot \boldsymbol{u}$ and rotations $\boldsymbol{e}_{k}=\boldsymbol{\theta} \times \boldsymbol{e}_{k}$ away from the static equilibrium. The incremental formulation [1,2] comprises above equations, linearized in the vicinity of a pre-stressed and deformed equilibrium state. The constraint (1) holds during the deformation, which relates displacements and small rotations $\boldsymbol{\theta}$ :

$$
\boldsymbol{u}^{\prime}=\varepsilon^{\cdot} \boldsymbol{t}+\boldsymbol{\theta} \times(1+\varepsilon) \boldsymbol{t}, \quad \varepsilon^{\cdot}=\boldsymbol{u}^{\prime} \cdot \boldsymbol{t} .
$$

The constitutive relations

$$
\begin{aligned}
Q_{t}^{\cdot} & =b \varepsilon^{*}, \\
\boldsymbol{M}^{*} & =\boldsymbol{\theta} \times \boldsymbol{M}+\mathbf{a} \cdot \boldsymbol{\theta}^{\prime}
\end{aligned}
$$

and the balance equations

$$
\begin{aligned}
& \boldsymbol{Q}^{\cdot \prime}+\boldsymbol{q}^{\cdot}=0, \\
& \boldsymbol{M}^{\cdot \prime}+\boldsymbol{u}^{\prime} \times \boldsymbol{Q}+\boldsymbol{r}^{\prime} \times \boldsymbol{Q}^{\cdot}+\boldsymbol{m} \cdot=0
\end{aligned}
$$

feature the force factors $\boldsymbol{Q}, \boldsymbol{M}$ in the rod in the previous equilibrium state and their small increments $\boldsymbol{Q}, \boldsymbol{M}$. Deriving Eqs. (12), we accounted for the variation of the stiffness tensor (9),

$$
\mathbf{a}^{\cdot}=a_{i j}\left(\boldsymbol{e}_{i} \boldsymbol{e}_{j}\right)^{\cdot}=\boldsymbol{\theta} \times \mathbf{a}-\mathbf{a} \times \boldsymbol{\theta},
$$

as well as for the Clebsch formula

$$
\boldsymbol{\kappa}^{\cdot}=\boldsymbol{\theta}^{\prime}+\boldsymbol{\theta} \times \boldsymbol{\kappa},
$$

see $[1,2]$ for details of derivation.

In Eulerian approach, stability of the static equilibrium of a conservative system is judged by the existence of infinitesimally close equilibrium configurations. It means that the configuration is critical if the above linear equations allow for a non-trivial solution $\boldsymbol{u}, \boldsymbol{\theta}, \boldsymbol{M}^{\boldsymbol{}}$ and $\boldsymbol{Q}^{\boldsymbol{}}$ with $\boldsymbol{q}^{\cdot}=0$ and $\boldsymbol{m}^{\boldsymbol{}}=0$; the boundary conditions are to be treated correspondingly. A pitchfork bifurcation of an equilibrium path corresponds to a classical buckling of a conservative system $[1,2,11]$.

\section{Statement of the problem}

We consider a flat strip of length $L$, which is clamped at both ends such that the distance between the ends is kinematically set to $H$. If $H<L$, then it is more than probable that the straight configuration of the strip becomes unstable and it bends out of its own plane. The deformed configuration would become essentially three-dimensional including torsion, if the undeformed strip is not straight and has a curvature radius $R$; see Fig. 2. According to the strategy declared above, we develop a rod model of the strip. Particles are associated by a material coordinate $s \in[-L / 2, L / 2]$ counted from the middle point of the strip, and the undeformed configuration has a curvature $R^{-1}$. The effect of the transverse gravity force is also accounted for in the analysis.

The possible deformed state, the global Cartesian coordinate system $x y z$ as well as the chosen orientation of the local basis vectors $\boldsymbol{e}_{k}$ are shown in Fig. 3 .

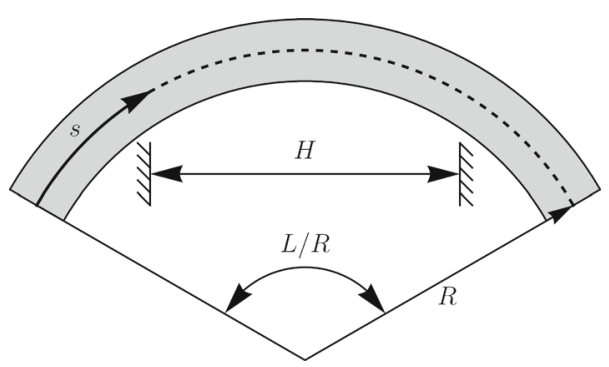

Fig. 2 Undeformed configuration of a flat strip with length $L$ and curvature radius $R$, which is clamped between points with a distance $H$ 


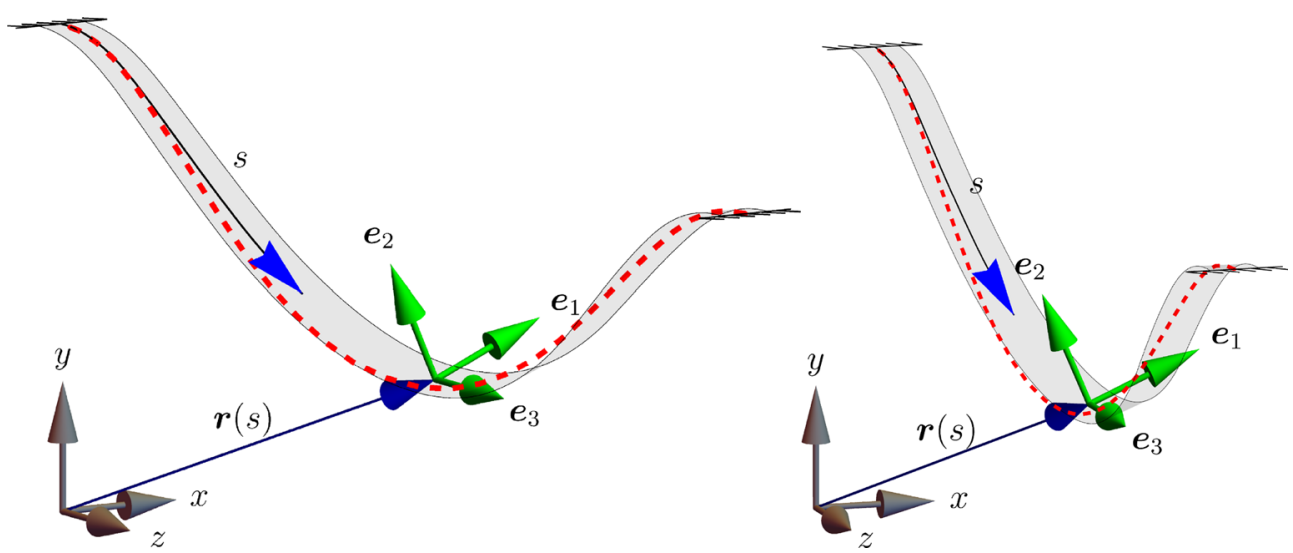

Fig. 3 Deformed strip with local basis, seen from two viewpoints; dashed line represents the plane configuration in the absence of the natural curvature at $R \rightarrow \infty$

The first local basis vector $\boldsymbol{e}_{1}$ is the direction in which the cross section of the rod is elongated; the corresponding bending stiffness $a_{11}$ is small. The second local basis vector $\boldsymbol{e}_{2}$ is orthogonal to the plane of the strip, and thus $\stackrel{\boldsymbol{e}}{2}_{2}$ is orthogonal to the plane of Fig. 2; the bending stiffness $a_{22}$ is high. The third local basis vector $\boldsymbol{e}_{3}$ is tangential to the deformed axis of the rod. Two variants of the clamping conditions are considered in the following (see also Fig. 4). The first option is to demand that both ends of the rod are directed along the horizontal line connecting them ( $z$ axis), such that

$$
\left.\left\{\boldsymbol{e}_{1}, \boldsymbol{e}_{2}, \boldsymbol{e}_{3}\right\}\right|_{s= \pm L / 2}=\{\boldsymbol{i}, \boldsymbol{j}, \boldsymbol{k}\},\left.\quad \boldsymbol{r}\right|_{s= \pm L / 2}= \pm H / 2 \boldsymbol{k}
$$

in which $\{\boldsymbol{i}, \boldsymbol{j}, \boldsymbol{k}\}$ are the unit basis vectors of the global Cartesian coordinates. The second considered option is vertical clamping, in which we demand

$$
\left.\left\{\boldsymbol{e}_{1}, \boldsymbol{e}_{2}, \boldsymbol{e}_{3}\right\}\right|_{s= \pm L / 2}=\{\boldsymbol{i}, \mp \boldsymbol{k}, \pm \boldsymbol{j}\},\left.\quad \boldsymbol{r}\right|_{s= \pm L / 2}= \pm H / 2 \boldsymbol{k} .
$$

In the absence of the natural curvature (when $R^{-1} \rightarrow 0$ ), the deformation takes place entirely in the plane $y z$. Increasing $R^{-1}$, we obtain symmetric configurations with displacements in $x$ direction and torsion. The semianalytical solution below accounts for the small natural curvature in terms of the incremental theory.



Fig. 4 In-plane deformed configurations of an elastica without gravity for various ratios of the length to the span $L / H$, horizontally clamped $(\alpha=0)$ on the left and vertically clamped $(\alpha=\pi / 2)$ on the right 


\section{Semi-analytical solution for an inextensible rod}

As long as $H<L$ and the gravity forces are moderate, we expect the effect of the tensional deformations to be negligible and first derive the equations under the assumption that $b \rightarrow \infty$ and $\varepsilon=0$. The geometrically nonlinear deformation in the plane $y z$, which takes place at no natural curvature, is then determined by the angle of rotation of particles from the straight undeformed state $\varphi(s)$ :

$$
\boldsymbol{r}(s)=y(s) \boldsymbol{j}+z(s) \boldsymbol{k}, \quad y^{\prime}=\sin \varphi, \quad z^{\prime}=\cos \varphi,
$$

see Fig. 4 for the examples of configurations, which take place at different ratios $L / H$ in the absence of gravity. We begin by integrating the first of the balance equations (7) for the force vector $Q$, which may not have transverse components in the middle point (at $s=0$ ) owing to the symmetry considerations:

$$
\boldsymbol{Q}^{\prime}-\rho g \boldsymbol{j}=0,\left.\quad \boldsymbol{Q}\right|_{s=0}=Q_{0} \boldsymbol{k} \Rightarrow \boldsymbol{Q}=Q_{0} \boldsymbol{k}+\rho_{s} \boldsymbol{j},
$$

$\rho$ is the mass density of the rod per unit length and $g$ is the free fall acceleration. Considering no external moment loading, $\boldsymbol{m}=0$, we substitute (19) in the balance of moments (second line in (7)) and with the kinematics (18) we find

$$
\boldsymbol{M}^{\prime}=\left(-Q_{0} \sin \varphi+\rho g s \cos \varphi\right) \boldsymbol{i}
$$

The differential equation for $\varphi$ follows as we compute the bending moment using the constitutive relations. There is no initial curvature about $x$ axis, $\Omega_{1}=0$, and with (3) we compute

$$
\kappa_{1}=\Omega_{1}=-\varphi^{\prime} .
$$

With the tensor of bending and torsional stiffness

$$
\mathbf{a}=a_{x} \boldsymbol{e}_{1} \boldsymbol{e}_{1}+a_{y} \boldsymbol{e}_{2} \boldsymbol{e}_{2}+a_{t} \boldsymbol{e}_{3} \boldsymbol{e}_{3}, \quad a_{11}=a_{x}, \quad a_{22}=a_{y}
$$

we find from (8) that

$$
\boldsymbol{M}=-a_{x} \varphi^{\prime} \boldsymbol{i},
$$

and the BVP follows from (20):

$$
\begin{aligned}
& a_{x} \varphi^{\prime \prime}-Q_{0} \sin \varphi+\rho g s \cos \varphi=0, \\
& \varphi(0)=0, \quad \varphi(L / 2)=\alpha, \quad \int_{0}^{L / 2} \cos \varphi \mathrm{d} s=H / 2 .
\end{aligned}
$$

The boundary condition at $s=0$ follows again from the symmetry, and both considered variants of the boundary conditions follow by either setting $\alpha=0$ (horizontal clamping) or $\alpha=\pi / 2$ (vertical one) for the value of $\varphi$ at the right end. The integral condition for the distance between the clamping points shall be satisfied by the appropriate choice of the horizontal force $Q_{0}$.

Particularly at higher ratios $L / H$, the solution of the BVP (24) is not unique: various equilibria are possible. Most of them are, however, unstable, see Levyakov and Kuznetsov [19], Singh and Goss [26] for detailed discussions. The most straightforward option for solving is the numerical integration of an initial value problem by choosing an initial approximation for the curvature in the middle $\varphi^{\prime}(0)$ as well as for the force $Q_{0}$ and then by iteratively seeking better approximations, which allow fulfilling the condition at $s=L / 2$ and the integral one from (24) in the framework of the Newton method. This, however, does not allow excluding unstable solutions from the consideration (in contrast to finite element schemes). Obtaining desired deformation forms with no loops as depicted in Fig. 4 is rather a matter of choice of the initial approximation for the Newton method. One may presume that the sought solutions correspond to the smallest $Q_{0}$ and $\varphi^{\prime}(0)$ (by absolute values), at least in the absence of the gravity loading. The numerical solution is easily implemented using Wolfram Mathematica ${ }^{1}$ and poses no difficulties as long as the bending stiffness is moderate. The problem becomes, however, ill-conditioned and challenging at very small bending stiffness, when very small variations in the initial conditions have enormous impact on the solution at $L / 2$.

\footnotetext{
${ }^{1}$ http://www.wolfram.com.
} 
Knowing the in-plane solution $\varphi(s)$, we consider its small increment because of the perturbation in the form of small natural curvature about the local axis $\boldsymbol{e}_{2}$,

$$
\stackrel{\Omega}{2}_{2}=R^{-1}
$$

For an inextensible rod Eq. (11) reduces to

$$
\boldsymbol{u}^{\prime}=\boldsymbol{\theta} \times \boldsymbol{t}
$$

Introducing components of the small rotation vector in the local basis and recalling that

$$
\boldsymbol{e}_{3}=\boldsymbol{t}=\boldsymbol{r}^{\prime}=\sin \varphi \boldsymbol{j}+\cos \varphi \boldsymbol{k}, \quad \boldsymbol{e}_{1}=\boldsymbol{i}, \quad \boldsymbol{e}_{2}=\boldsymbol{e}_{3} \times \boldsymbol{e}_{1}=\cos \varphi \boldsymbol{j}-\sin \varphi \boldsymbol{k},
$$

we write

$$
\boldsymbol{\theta}=\theta_{k} \boldsymbol{e}_{k}, \quad \boldsymbol{u}^{\prime}=\theta_{2} \boldsymbol{e}_{1}-\theta_{1} \boldsymbol{e}_{2} .
$$

The distributed gravity force does not change, $\boldsymbol{q}^{\cdot}=0$, and from the first line in (13) as well as symmetry considerations we conclude that $\boldsymbol{Q}^{\cdot}=Q_{0} \boldsymbol{i}=$ const.

Now, we extend the kinematic Clebsch formula for the increment of the strain measure $\boldsymbol{\kappa}^{\cdot}(15)$ : the imposed natural curvature of the rod results in an additional term $-\AA_{2}^{\circ} \dot{e}_{2}$ on the right-hand side (compare to the definition of $\boldsymbol{\kappa}$ in (5)). Thus we obtain an extended version of the incremental constitutive relation for the moment in the second line of (12):

$$
\begin{aligned}
\boldsymbol{M}= & \boldsymbol{\theta} \times \boldsymbol{M}+\mathbf{a} \cdot \boldsymbol{\theta}^{\prime}-a_{y} \check{\Omega}_{2} \dot{\boldsymbol{e}}_{2}=a_{x} \varphi^{\prime}\left(\theta_{2} \boldsymbol{e}_{3}-\theta_{3} \boldsymbol{e}_{2}\right) \\
& +a_{x} \theta_{1}^{\prime} \boldsymbol{e}_{1}+a_{y} \theta_{2}^{\prime} \boldsymbol{e}_{2}+a_{t} \theta_{3}^{\prime} \boldsymbol{e}_{3}+\varphi^{\prime}\left(a_{y} \theta_{3} \boldsymbol{e}_{2}-a_{t} \theta_{2} \boldsymbol{e}_{3}\right)-a_{y} \check{\Omega}_{2} \dot{\boldsymbol{e}}_{2},
\end{aligned}
$$

we substituted the bending moment for the pre-deformed state (23) and took into account that $\boldsymbol{e}_{2}^{\prime}=-\varphi^{\prime} \boldsymbol{e}_{3}$, $\boldsymbol{e}_{3}^{\prime}=\varphi^{\prime} \boldsymbol{e}_{2}$.

Finally, we compute the derivative $\boldsymbol{M}^{\prime \prime}$, substitute into the incremental equation of balance of moments (second line of (13)) with $\boldsymbol{m}^{\cdot}=0$ and project the resulting vectorial differential equation for the three components of $\boldsymbol{\theta}$ on the directions of the local basis $\boldsymbol{e}_{k}$. The mathematical transformations are easy with a computer algebra software, and in the end of the day we arrive at a system of differential equations for the components $\theta_{k}$. The first one (projection on the direction $\boldsymbol{e}_{1}$ ) reads

$$
a_{x} \theta_{1}^{\prime \prime}-\left(Q_{0} \cos \varphi+\rho g s \sin \varphi\right) \theta_{1}+Q_{0}^{\cdot} \sin \varphi=0 .
$$

The problem for the increment of the deformation in the plane $y z$ decouples, and along with the trivial boundary conditions for $\theta_{1}$ as well as the requirement of vanishing increment of the integral conditions in (24) this allows us to conclude that $\theta_{1}=0$ identically and that $Q_{0}^{*}=0$.

Projections of the vectorial balance equations on the directions $\boldsymbol{e}_{2}$ and $\boldsymbol{e}_{3}$ result in a system of equations for the torsional and out-of-plane bending components of $\boldsymbol{\theta}$ :

$$
\begin{aligned}
a_{y} \theta_{2}^{\prime \prime}+ & \left(a_{t}+a_{y}-a_{x}\right) \varphi^{\prime} \theta_{3}^{\prime}+\left(a_{y}-a_{x}\right) \varphi^{\prime \prime} \theta_{3} \\
& -\left(\left(a_{t}-a_{x}\right) \varphi^{\prime 2}+Q_{0} \cos \varphi+\rho g s \sin \varphi\right) \theta_{2}=0, \\
a_{t} \theta_{3}^{\prime \prime}- & \left(a_{t}+a_{y}-a_{x}\right) \varphi^{\prime} \theta_{2}^{\prime}-\left(a_{y}-a_{x}\right) \varphi^{\prime 2} \theta_{3} \\
& -\left(\left(a_{t}-a_{x}\right) \varphi^{\prime \prime}+Q_{0} \sin \varphi-\rho g s \cos \varphi\right) \theta_{2}+a_{y} \varphi^{\prime} \AA_{2} \cdot{ }_{2}=0 .
\end{aligned}
$$

The linear BVP for the components $\theta_{2}$ and $\theta_{3}$ follows from Eq. (31) with the boundary conditions

$$
\begin{aligned}
& \theta_{2}(0)=0, \quad \theta_{2}(L / 2)=0, \\
& \theta_{3}^{\prime}(0)=0, \quad \theta_{3}(L / 2)=0,
\end{aligned}
$$

as the strip is clamped at the right end and the solution is symmetric at $s=0$.

The second equation in (31) becomes slightly shorter if we exclude the constant $Q_{0}$ using the equation of in-plane bending (24):

$$
\begin{aligned}
a_{y} \theta_{2}^{\prime \prime} & +\left(a_{t}+a_{y}-a_{x}\right) \varphi^{\prime} \theta_{3}^{\prime}+\left(a_{y}-a_{x}\right) \varphi^{\prime \prime} \theta_{3} \\
& -\left(\left(a_{t}-a_{x}\right) \varphi^{\prime 2} \sin \varphi+\rho g s+a_{x} \varphi^{\prime \prime} \cos \varphi\right) \theta_{2} / \sin \varphi=0, \\
a_{t} \theta_{3}^{\prime \prime} & -\left(a_{t}+a_{y}-a_{x}\right) \varphi^{\prime} \theta_{2}^{\prime}-\left(a_{y}-a_{x}\right) \varphi^{\prime 2} \theta_{3}-a_{t} \varphi^{\prime \prime} \theta_{2}+a_{y} \varphi^{\prime} \stackrel{\Omega}{2}_{2}=0 .
\end{aligned}
$$


The absence of $Q_{0}$ simplifies the logic of solution. Although the coefficient with $(\cdots) / \sin \varphi$ in the first equation will not be singular as $\varphi$ fulfills (24), it needs to be treated in the numerical procedure, e.g., by shifting the boundary conditions (32) from $s=0$ to a neighboring point $s=\delta$, in the computations below we used $\delta=10^{-6} L$.

Comparing solutions in the numerical examples below, we use the rotation of the middle point of the strip $\theta_{3}(0)$ as well as its lateral deflection

$$
u_{x}(0)=-\int_{0}^{L / 2} \theta_{2} \mathrm{~d} s
$$

as principal kinematic entities.

\section{Asymptotics for a thin strip}

Flat strips with thin cross sections are very stiff for bending about the axis $\boldsymbol{e}_{2}$; thus $a_{y} \gg a_{x}, a_{t}$ holds. In this section we seek equations for the limiting case, when this stiffness is very high, and introduce a formal small parameter $\lambda$ replacing in the equations $a_{y}$ by $\lambda^{-1} a_{y}$. The unknown functions are sought in the form of formal asymptotic expansions,

$$
\theta_{2}=\lambda^{0} \stackrel{\theta}{\theta}_{2}+\lambda^{1} \hat{\theta}_{2}^{1}+\cdots, \quad \theta_{3}=\lambda^{0} \theta_{3}^{0}+\lambda^{1}{ }^{1} \theta_{3}+\cdots .
$$

Now we formally substitute the above series in the system (33) and balance terms of the order $\lambda^{-1}$, thus obtaining equations for the leading order terms:

$$
\begin{aligned}
& 0_{2}^{\prime \prime}+\varphi^{\prime} \theta_{3}^{\prime}+\varphi^{\prime \prime}{ }^{0} \theta_{3}=0, \\
& { }_{\theta_{2}^{\prime}}^{\prime}+\varphi^{\prime}{ }_{3}^{0}-\stackrel{\circ}{\Omega}_{2}^{\circ}=0 .
\end{aligned}
$$

The first equation is evidently a derivative of the second one, and at the first step we find

$$
\stackrel{0}{\theta}_{3}=\left(\stackrel{\circ}{\Omega}_{2}-\stackrel{0}{\theta_{2}^{\prime}}\right) / \varphi^{\prime} .
$$

We are not able to compute the leading order terms yet. The second step of the procedure of asymptotic splitting (see $[1,2,27]$ for discussions) is required, which allows finding the principal terms from the conditions of solvability for the minor ones. We again address the full system (33) and balance terms of the order $\lambda^{0}$, taking (37) into account. The resulting equations for the first-order correction terms $\stackrel{1}{\theta}_{2,3}$ again have the structure of the system (36), but are no longer homogeneous as the leading order terms are present. Equations need to be non-contradictory (i.e., solvable), and we finally arrive at a single fourth-order differential equation for the $\theta_{2}$ :

$$
\begin{aligned}
& a_{t} \varphi^{\prime 3}{ }_{0}^{0}{ }_{2}^{I V}-4 a_{t} \varphi^{\prime 2} \varphi^{\prime \prime} \theta_{2}^{\prime \prime \prime}+\left(\left(2 a_{t}+a_{x}\right) \varphi^{\prime 4}+8 a_{t} \varphi^{\prime \prime 2}-3 a_{t} \varphi^{\prime} \varphi^{\prime \prime \prime}\right) \varphi^{\prime} \theta_{2}^{\prime \prime} \\
& \quad+\left(\varphi^{\prime \prime}\left(2 a_{x} \varphi^{4}-8 a_{t} \varphi^{\prime \prime 2}+7 a_{t} \varphi^{\prime} \varphi^{\prime \prime \prime}\right)-a_{t} \varphi^{\prime 2} \varphi^{I V}\right) \hat{\theta}_{2}^{\prime} \\
& \quad+\left(\left(a_{t}+a_{x}\right) \varphi^{\prime 4}+\left(a_{t}+2 a_{x}\right)\left(\varphi^{\prime} \varphi^{\prime \prime \prime}-\varphi^{\prime \prime 2}\right)+\left(\rho g s+a_{x} \varphi^{\prime \prime} \cos \varphi\right) \varphi^{\prime 2} / \sin \varphi\right) \varphi^{\prime 3} \theta_{2}^{0} \\
& \quad+a_{t}\left(\varphi^{\prime \prime}\left(\varphi^{\prime 4}+8 \varphi^{\prime \prime 2}-7 \varphi^{\prime} \varphi^{\prime \prime \prime}\right)+\varphi^{\prime 2} \varphi^{I V}\right) \stackrel{\Omega}{\Omega}_{2}=0 .
\end{aligned}
$$

Although not compact, the single equation for the principal term in $\theta_{2}$ is more efficient when $a_{y}$ is so large that the original system (33) becomes ill-conditioned. The boundary conditions for (38) read

$$
\stackrel{0}{\theta}_{2}(0)=0, \quad \stackrel{0}{\theta}_{2}(L / 2)=0, \quad \stackrel{0}{\theta}_{2}^{\prime \prime}(0)=0, \quad \stackrel{0}{\theta}_{2}^{\prime}(L / 2)=\stackrel{\circ}{\Omega}_{2},
$$

the latter two follow from the conditions for $\theta_{3}$ in (32) and the relation (37) together with $\varphi^{\prime \prime}(0)=0$ from (24).

Finally we notice that the derived asymptotics is valid as long as the in-plane deformed configuration has no inflexion points, in which the curvature changes its sign and $\varphi^{\prime}$ vanishes. Such points are highly probable 
in the case of horizontal clamping conditions (16) with $\alpha=0$ in (24). The relation (37) cannot be written in the vicinity of such points, and the required analysis becomes much more complicated. Therefore, in the numerical section below we apply the asymptotic solution only to the case of vertical clamping and as long as the span $H$ is sufficiently high, such that the curvature $\varphi^{\prime}$ remains positive.

\section{Numerical simulations for an inextensible strip}

For benchmark problems, we adopted the bending and torsional stiffness values as well as linear density of the rod model to be

$$
a_{x}=E \frac{w h^{3}}{12}, \quad a_{y}=E \frac{h w^{3}}{12}, \quad a_{t}=\frac{E}{2(1+v)} \frac{w h^{3}}{3}, \quad \rho=\rho_{3} w h .
$$

The numerical values for the width of the cross section $w$, its thickness $h$, Young's modulus $E$, Poisson's ratio $v$, material density $\rho_{3}$, free fall acceleration $g$ and material length $L$ are chosen as

$$
\begin{array}{ll}
w=0.01 \beta, & h=0.002 / \beta, \quad E=2.1 \cdot 10^{11}, \quad v=0.3, \\
\rho_{3}=7800, \quad g=9.8, \quad L=1 .
\end{array}
$$

All numerical values here and below are written using the SI system of units. Varying $\beta$ we change the shape of the cross section. In the following, we compare solutions for a thick $(\beta=1, w / h=5)$ and a thin $(\beta=4$, $w / h=80)$ strip. The length of the span $H$ as well as the type of the boundary conditions determined by the angle $\alpha$ shall be varied.

We also solved the problem using the shell finite element formulation, presented in [23]. Comparison between rod and shell solutions allows understanding the range of applicability of the rod model for the considered sort of problems. The shell model featured a mesh of $2 \times 60$ finite elements with bi-cubic approximation. The equivalent kinematic variables used for comparison (rotation and deflection in the middle) were estimated using linear regression of the nodal positions of the deformed configuration of the shell, which for $H=0.8$, the imperfection value $\Omega_{2}=0.15$ (corresponds to $R \approx 6.67$ ) and horizontal clamping is demonstrated in Fig. 5 for the case of a thin cross section. This solution with significant out-of-plane deformations is still almost linear with respect to the imperfection: reducing $\Omega_{2}$ by a factor 100 and correspondingly scaling the computed out-of-plane kinematic variables, we observe ca. $0.15 \%$ change in the rotation angle $\theta_{3}(0)$ of the middle cross section $s=0$, and its out-of-plane deflection $u_{x}(0)$ changes by ca. $0.04 \%$.

Having stated that, for all subsequent solutions we use a very small value $\Omega_{2}^{\cdot}=10^{-4}$, and all the presented deflections and rotations are scaled correspondingly such that they correspond to the unit imperfection (the computed values are divided by $\Omega_{2}^{\cdot}=10^{-4}$ ). We also notice here that the presented rod solutions perfectly correspond to the converged results of equivalent rod finite element simulations featuring classical rod theory $[20,21]$ as the effect of extensibility in the considered case is very small.

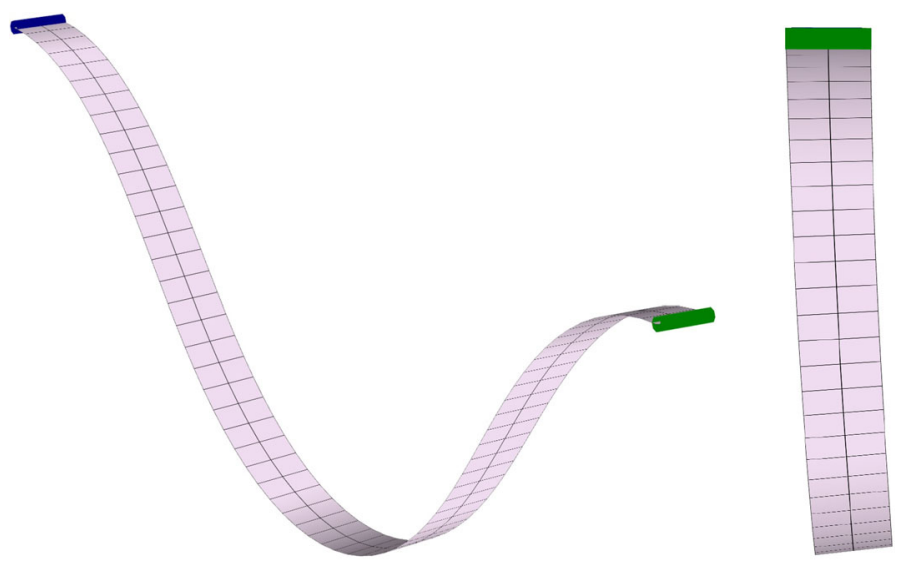

Fig. 5 Deformed shell: horizontal clamping, thin strip, $H=0.8, \AA_{2}^{\cdot}=0.15$, isometric and front views 
Table 1 Comparison of lateral deflections resulting from the full and the asymptotically reduced BVPs

\begin{tabular}{lcccc}
\hline$H$ & $u_{x}(0)$, thick & 0 & $u_{x}(0)$, thin & 0 \\
\hline 0.72 & -0.01820 & -0.01754 & -0.01851 & -0.01848 \\
0.79 & 0.00030 & 0.00322 & 0.00140 & 0.00149 \\
0.86 & 0.02257 & 0.03377 & 0.03053 & 0.03070 \\
\hline
\end{tabular}

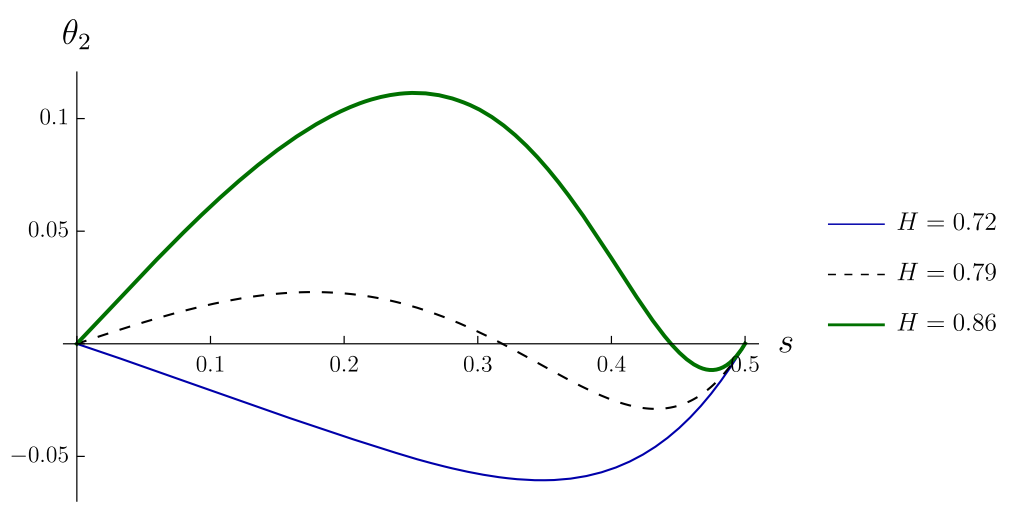

Fig. 6 Angle of rotation $\theta_{2}$ for the rod model of a thick strip with vertical clamping for various spans

The lateral deflections, computed for the thick and for the thin vertically clamped strips using the full BVP (32), (33) as well as for the asymptotically reduced one (38), (39) are compared in Table 1 for different span lengths $H$. As expected, the asymptotic solution is evidently much more accurate for the thin strip with its very high out-of-plane bending stiffness $a_{y}$ compared to $a_{x}$ and $a_{t}$. For the thick strip, the asymptotic solution becomes more accurate at lower $H$ : for $H=0.6$ its relative error is $0.3 \%$. We also notice that the lateral deflection changes its sign as the clamped ends are moved. To clarify the effect, in Fig. 6 we compare the computed distributions of the angle of rotation $\theta_{2}$ along the axis of the strip. For small $H$ the angle remains always negative and from (34) we find positive deflections $u_{x}(0)$. At higher $H$ we obtain sign-changing $\theta_{2}(s)$, which eventually changes the sign of the integral in (34).

Comparison against shell solutions is provided in Fig. 7. For the case of the vertical clamping, we computed the rotation and the lateral deflection of the middle cross section $s=0$ of the thin and of the thick strips in dependence on the span length using both, the rod and the shell models. The first observation is that all curves are very close for moderately small $H$, when the rod solution is close to the asymptotic one. Indeed, the unperturbed solution $\varphi(s)$ is very similar for both the thin and the thick strips as the influence of gravity is low, and from the asymptotic equation (38) we see that the result is determined by the ratio $a_{x} / a_{t}$, which is the same for both cases. Increasing $H$, we see that the behavior of the thin and the thick strips is qualitatively different. The good correspondence to the shell solution holds in a broader range of $H$ for the thick strip, and the rod kinematics apparently becomes insufficient to describe the complicated three-dimensional behavior of a thin one when $H / L$ approaches 1 and the curvature of the strip is growing near the end points because of the vertical clamping. Examining the shell solution, we indeed observe that the cross sections of the strip significantly deform in the regions with high curvature $\varphi^{\prime}$ : the originally straight transverse fibers become S-shaped, which cannot be reproduced by the rod model.

Finally we consider the case of horizontal clamping and compare solutions for thick and thin strips, with and without gravity in both shell and rod models in Fig. 8. Comparing the thick dashed line (rod thick with gravity) against Fig. 7, we see the effect of the boundary conditions: the values of $u_{x}(0)$ are similar only at small $H$. The gravity force reduces the lateral deflection, and the accuracy of the rod solution against the shell one is comparable to that in Fig. 7. Moreover, this time the rotation angle $\theta_{3}(0)$ in the rod and the shell solutions is much closer than in the case of the vertical clamping as no zones of high curvature develop when $H$ is growing. 

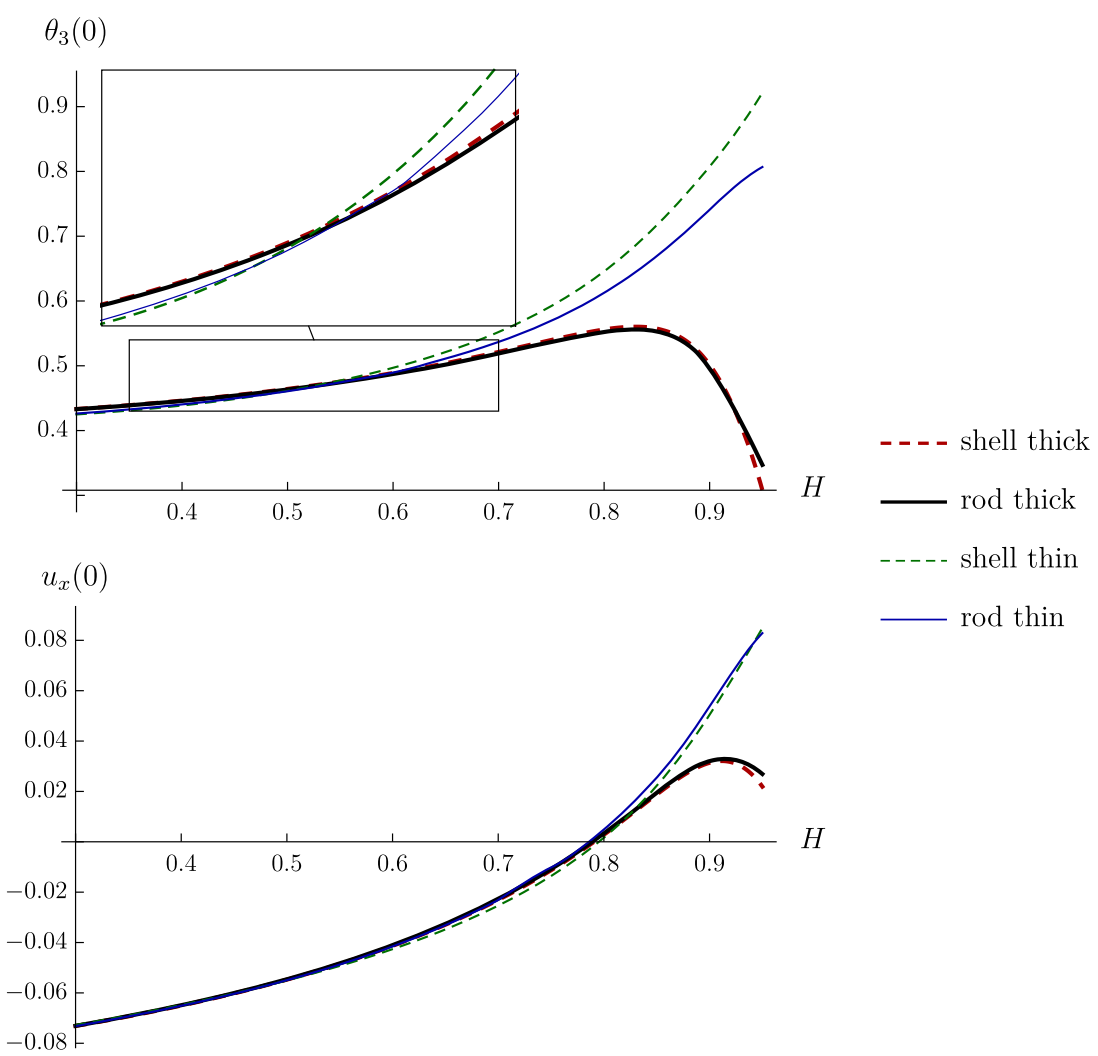

-..-- shell thin

Fig. 7 Comparison of rod and shell solutions for the vertically clamped strip

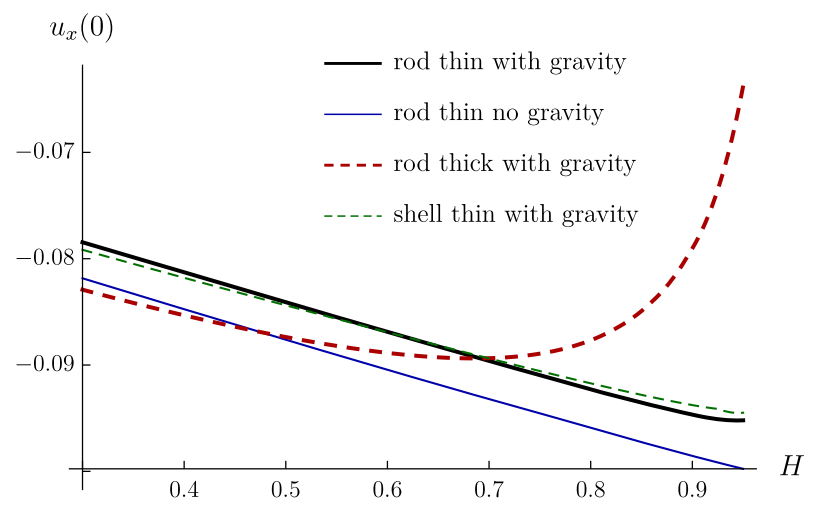

Fig. 8 Comparison of the lateral deflection of the middle point for the horizontally clamped strip in various settings

\section{Special case: semicircle}

The derived equations and their solution become remarkably simple in the particular case, when the curvature of the in-plane pre-deformed state is constant, $\varphi^{\prime}=$ const. We consider a beam with vertical clamping conditions with $H=2 / \eta$ and $L=\pi / \eta, \eta$ being the curvature of the resulting semicircle. Considering the case with no gravity, $g=0$, we see that the BVP (24) is fulfilled by $\varphi=\eta s, Q_{0}=0$. This is a pure bending solution with $\kappa_{1}=-\eta$ and $M_{1}=-a_{x} \eta$. Again we apply a small natural curvature $\Omega_{2}^{\prime}$ about the local axis $\mathbf{e}_{2}$. The BVP for the small rotations $\theta_{2}$ and $\theta_{3}(33)$ becomes

$$
\begin{aligned}
& a_{y} \theta_{2}^{\prime \prime}+\eta\left(a_{t}+a_{y}-a_{x}\right) \theta_{3}^{\prime}-\eta^{2}\left(a_{t}-a_{x}\right) \theta_{2}=0, \\
& a_{t} \theta_{3}^{\prime \prime}-\eta\left(a_{t}+a_{y}-a_{x}\right) \theta_{2}^{\prime}-\eta^{2}\left(a_{y}-a_{x}\right) \theta_{3}=-\eta a_{y} \Omega_{2}, \\
& \theta_{2}(0)=0, \quad \theta_{2}(L / 2)=0, \quad \theta_{3}^{\prime}(0)=0, \quad \theta_{3}(L / 2)=0 .
\end{aligned}
$$


Table 2 Small rotation of the middle point $\theta_{3}(0)$, established with different methods

\begin{tabular}{lr}
\hline Asymptotic with $\Omega_{1}^{\circ}=-\eta$ & $\theta_{3}(0)$ \\
Asymptotic with $\Omega_{1}=0$ & 0.00163662 \\
Shell model with $\Omega_{1}=0$ & 0.00160170 \\
Exact with $\Omega_{1}=0$ & 0.00155358 \\
Exact with $\Omega_{1}=-\eta$ & 0.00155226 \\
\hline
\end{tabular}

The differential equation has constant coefficients, and the solution to this BVP reads

$$
\begin{aligned}
\theta_{2} & =\frac{\sin (\eta \zeta s)-\sin (\eta s) \sin (\zeta \pi / 2)}{\eta \zeta \cos (\zeta \pi / 2)} \AA_{2} \text { with } \zeta^{2}=\frac{\left(a_{y}-a_{x}\right)\left(a_{t}-a_{x}\right)}{a_{y} a_{t}}, \\
\theta_{3} & =\left(\frac{a_{y}}{a_{y}-a_{x}} \cos \frac{\zeta \pi}{2}-\frac{a_{y}}{a_{y}-a_{x}} \cos (\eta \zeta s)+\frac{1}{\zeta} \cos (\eta s) \sin \frac{\zeta \pi}{2}\right) \frac{\stackrel{\circ}{2}_{2}}{\eta \cos (\zeta \pi / 2)} .
\end{aligned}
$$

The parameter $\zeta$ is real for thin rectangular cross sections with stiffness values (40) with $v<1 / 2$ as

$$
a_{t}>E w h^{3} / 9>a_{x},
$$

whereas $a_{y}>a_{x}$ is trivial. Because the strip is thin compared to its width, we show the asymptotic formulas by considering $a_{x, t} / a_{y} \rightarrow 0$ :

$$
\begin{aligned}
& \stackrel{0}{\theta}_{2}=\frac{\sin (\eta \gamma s)-\sin (\eta s) \sin (\gamma \pi / 2)}{\eta \zeta \cos (\gamma \pi / 2)} \stackrel{\circ}{\Omega}_{2}, \\
& \stackrel{0}{\theta}_{3}=R\left(\stackrel{\Omega}{\Omega}_{2}-\stackrel{0}{\theta}_{2}^{\prime}\right), \quad \gamma^{2}=\lim _{a_{y} \rightarrow \infty} \zeta^{2}=\frac{a_{t}-a_{x}}{a_{t}},
\end{aligned}
$$

which is slightly simpler than the exact solution. It is also possible to use the above asymptotic equations to obtain this result.

The solution becomes even more simple if the rod is initially curved in the plane $y z$ such, that the semicircular configuration is stress free and the incremental formulation becomes identical to the equations of the linear theory. Indeed, considering a natural curvature $\Omega_{1}=-\eta$, we find $\kappa_{1}=0$ and $M_{1}=0$. The resulting exact equations are (42) with omitted $a_{x}$. The solution reads

$$
\begin{aligned}
\theta_{2} & =-\frac{2}{\pi} s \cos (\eta s) \stackrel{\Omega}{2}_{2}, \\
\theta_{3} & =\frac{2\left(a_{y}-a_{t}\right) \cos (\eta s)+\left(a_{y}+a_{t}\right)(\pi R-2 \eta s \sin (\eta s))}{\pi \eta\left(a_{y}+a_{t}\right)} \stackrel{\Omega}{2}_{2} .
\end{aligned}
$$

We see that $\theta_{2}$ does not depend on the properties of the cross section and the asymptotic result would therefore be the same as the exact one - which, however, is not the case for the angle of twisting $\theta_{3}$.

Finally we compare different solutions for $\eta=1, w=0.01, h=0.002$ and $\Omega_{2}=10^{-3}$. The computed values of $\theta_{3}(0)$ are presented in Table 2 . While both the rod and the shell solutions of the original problem are remarkably close, the error introduced by neglecting the pre-stress (exact solution with $\Omega_{1}=-\eta$ ) is below $1 \%$. The asymptotic solutions are less accurate in this case of a thick strip with $w / h=5$.

\section{High pre-tension of the extensible strip}

The problem at hand is important for the study of such practically relevant phenomena as the lateral run-off of the flat belt in a belt drive or formation of waves in a flat metal strip within a rolling mill. Both cases differ from the above examples by the presence of a high tension force, such that the extension of the strip cannot be neglected. We consider a horizontally clamped rod, and instead of (24) the plane bending problem is now governed by the following BVP:

$$
\begin{aligned}
& a_{x} \varphi^{\prime \prime}+(1+\varepsilon)\left(-Q_{0} \sin \varphi+\rho g s \cos \varphi\right)=0, \\
& \varphi(0)=0, \quad \varphi(L / 2)=0, \quad \int_{0}^{L / 2}(1+\varepsilon) \cos \varphi \mathrm{d} s=H / 2 .
\end{aligned}
$$

The axial strain follows with the tangential force: 


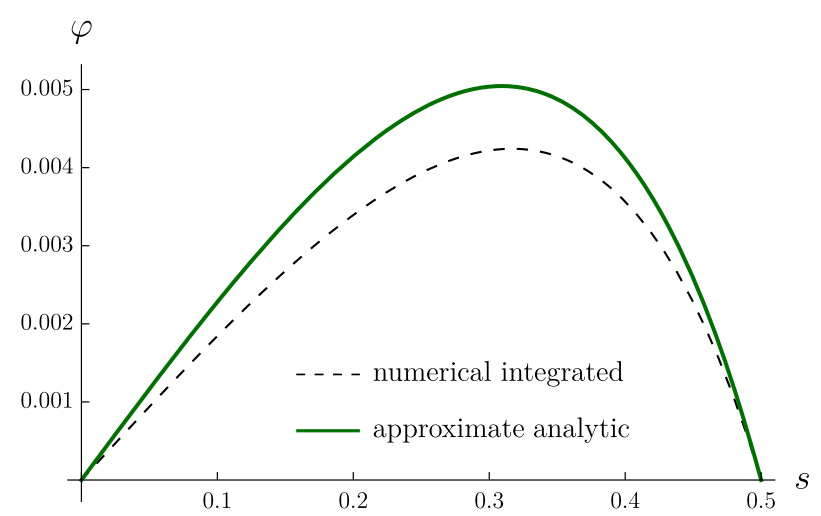

Fig. 9 Numerical and analytical solutions of the BVP (49) for a weakly pre-tensioned strip with $\varepsilon_{0}=10^{-5}$

$$
b \varepsilon=Q_{t}=Q_{0} \cos \varphi+\rho g s \sin \varphi .
$$

At high pre-tension, the strip behaves like a string inside the domain, and narrow edge layers with bending effects develop near the boundaries, which makes the problem ill-conditioned and difficult to treat with the shooting method. However, small deflections allow constructing an approximate analytical solution. Without gravity the strip is straight, spanned with $H=\left(1+\varepsilon_{0}\right) L, \varepsilon_{0}>0$, and the axial force is $Q_{0}=b \varepsilon_{0}$. Because of small deflections, we assume small angles and both $Q_{0}$ and $\varepsilon=\varepsilon_{0}$ remaining constant. We obtain

$$
a_{x} \varphi^{\prime \prime}+\left(1+\varepsilon_{0}\right)\left(\rho g s-b \varepsilon_{0} \varphi\right)=0, \quad \varphi(0)=0, \quad \varphi(L / 2)=0,
$$

with the solution

$$
\varphi=\frac{\rho g\left(L e^{\xi(L-2 s) / 2}-L e^{\xi(L+2 s) / 2}+2 s e^{L \xi}-2 s\right)}{2 b \varepsilon_{0}\left(e^{L \xi}-1\right)}, \quad \xi^{2}=\frac{\left(1+\varepsilon_{0}\right) b \varepsilon_{0}}{a_{x}} .
$$

We use the parameters (41) for a thick strip, setting for simplicity $v=0$, and test the solution (50) against the numerical solution of the BVP (49). While for $\varepsilon_{0}=10^{-4}$, the maximal relative difference in the values of $\varphi$ does not exceed $0.2 \%$, for the lower pre-stretch $\varepsilon_{0}=10^{-5}$ the error reaches $18.5 \%$, see Fig. 9 .

Finally we consider the incremental problem with the small natural curvature $\Omega_{2}^{0}=10^{-3}$ (again, the results below are scaled by this value of the imperfection). The counterpart to system (31) for the case of extensible rods contains the factor $1+\varepsilon$ in front of the terms with $Q_{0}$ and $\rho g$; we do not present the derivation here, as it is methodologically very similar to the one in Sect. 4. We solve the equations setting $\varepsilon=\varepsilon_{0}$ and then use $u_{x}^{\prime}=\left(1+\varepsilon_{0}\right) \theta_{2}$ as a counterpart to (34) to find the lateral deflection. For two values of the pre-stretch, we compare the computation results against equivalent rod (unshearable, but extensible) and shell finite element simulations in Table 3. Moreover, we also present the values, which result from the linear incremental equations for the inextensible case (31) as if $\varepsilon=0$ (the analytic expression for $\varphi(s)(50)$ was used in both incremental solutions).

Table 3 Lateral deflection and rotation of the middle cross section of the strip depending on the pre-stretch computed with rod and shell models

\begin{tabular}{llll}
\hline Model & $\varepsilon_{0}$ & $\theta_{3}(0)$ & $u_{x}(0)$ \\
\hline Rod, incremental & $10^{-4}$ & $4.375 \cdot 10^{-3}$ & $-3.637 \cdot 10^{-7}$ \\
Rod, finite elements & & $4.363 \cdot 10^{-3}$ & $-3.612 \cdot 10^{-7}$ \\
Shell, finite elements & & $4.358 \cdot 10^{-3}$ & $-3.608 \cdot 10^{-7}$ \\
Rod, incremental, $\varepsilon=0$ & & $4.312 \cdot 10^{-3}$ & $-3.533 \cdot 10^{-7}$ \\
Rod, incremental & $10^{-3}$ & $5.272 \cdot 10^{-4}$ & $-1.285 \cdot 10^{-9}$ \\
Rod, finite elements & & $5.272 \cdot 10^{-4}$ & $-1.284 \cdot 10^{-9}$ \\
Shell, finite elements & & $5.222 \cdot 10^{-4}$ & $-1.273 \cdot 10^{-9}$ \\
Rod, incremental, $\varepsilon=0$ & $5.182 \cdot 10^{-4}$ & $-1.242 \cdot 10^{-9}$ \\
\hline
\end{tabular}


The perfect correspondence to the rod finite element solution at high pre-stretch justifies the assumptions of the semi-analytic model. The approximate expression for $\varphi(s)$ is not so accurate at lower $\varepsilon_{0}$, which determines the differences between the values in the first and the second lines of the upper block of the table. High pre-tension reduces the lateral deformations, but the rotational response to the imperfection is still significant: the intrinsic curvature makes one edge of the strip longer than the other one, and the response to the gravity loading becomes unsymmetric.

Predictions of the rod and of the shell models of the strip remain close, which validates the use of the computationally more efficient one-dimensional model to the analysis of the above-mentioned technical problems. This observation provides a practically relevant dimension to the considered academic example of nonlinear spatial deformations of curved elastic strips.

Acknowledgements Open access funding provided by TU Wien (TUW). This research was supported by the Austrian Research Promotion Agency (FFG), project number: 861493.

Open Access This article is distributed under the terms of the Creative Commons Attribution 4.0 International License (http:// creativecommons.org/licenses/by/4.0/), which permits unrestricted use, distribution, and reproduction in any medium, provided you give appropriate credit to the original author(s) and the source, provide a link to the Creative Commons license, and indicate if changes were made.

\section{References}

1. Eliseev, V.V.: Mechanics of Deformable Solid Bodies. St. Petersburg State Polytechnical University Publishing House, St. Petersburg (2006). (in Russian)

2. Vetyukov, Y.: Nonlinear Mechanics of Thin-Walled Structures. Asymptotics, Direct Approach and Numerical Analysis. Foundations of Engineering Mechanics. Springer, Vienna (2014). https://doi.org/10.1007/978-3-7091-1777-4

3. Huynen, A., Detournay, E., Denoël, V.: Eulerian formulation of elastic rods. Proc. R. Soc. A 472, 1-23 (2016)

4. Belyaev, A.K., Eliseev, V.V.: Flexible rod model for the rotation of a drill string in an arbitrary borehole. Acta Mech. 229, 841-848 (2018)

5. Vetyukov, Y.: Stability and supercritical deformation of a circular ring with intrinsic curvature. In: Irschik, H., Belyaev, A., Krommer, M. (eds.) Dynamics and Control of Advanced Structures and Machines, pp. 23-32. Springer, Berlin (2017)

6. Dias, M.A., Audoly, B.: A non-linear rod model for folded elastic strips. J. Mech. Phys. Solids 62, 57-80 (2014)

7. Audoly, B., Seffen, K.: Buckling of naturally curved elastic strips: the ribbon model makes a difference. J. Elast. 119, 293-320 (2015)

8. Fang, J., Chen, J.-S.: Deformation and vibration of a spatial elastica with fixed end slopes. Int. J. Solids Struct. 50, 824-831 (2013)

9. Simitses, G.J., Hodges, D.H.: Fundamentals of Structural Stability. Elsevier, New York (2006)

10. Glavardanov, V.B., Maretic, R.B.: Stability of a twisted and compressed clamped rod. Acta Mech. 202, 17-33 (2009)

11. Ziegler, H.: Principles of Structural Stability, 2nd edn. Birkhäuser Verlag, Basel (1977)

12. Goriely, A.: Twisted elastic rings and the rediscoveries of Michell's instability. J. Elast. 84, 281-299 (2006)

13. Vetyukov, Yu.: Direct approach to elastic deformations and stability of thin-walled rods of open profile. Acta Mech. 200(3-4), $167-176(2008)$

14. Vetyukov, Y., Oborin, E., Scheidl, J., Krommer, M., Schmidrathner, C.: Flexible belt hanging on two pulleys: contact problem at non-material kinematic description. Int. J. Solids Struct. (submitted)

15. Belyaev, A.K., Eliseev, V.V., Irschik, H., Oborin, E.A.: Contact of two equal rigid pulleys with a belt modelled as Cosserat nonlinear elastic rod. Acta Mech. 228, 4425-4434 (2017)

16. Kong, L., Parker, R.G.: Steady mechanics of belt-pulley systems. ASME J. Appl. Mech. 72, 25-34 (2005)

17. Denoël, V., Detournay, E.: Eulerian formulation of constrained elastica. Int. J. Solids Struct. 48, 625-636 (2011)

18. Humer, A.: Exact solutions for the buckling and postbuckling of shear-deformable beams. Acta Mech. 224, 1493-1525 (2013)

19. Levyakov, S.V., Kuznetsov, V.V.: Stability analysis of planar equilibrium configurations of elastic rods subjected to end loads. Acta Mech. 211, 73-87 (2010)

20. Vetyukov, Yu.M., Eliseev, V.V.: Modeling of building frames as spatial rod structures with geometric and physical nonlinearities (in Russian). Comput. Contin. Mech. 3(3), 32-45 (2010). http://www.icmm.ru/journal/download/CCMv3n3a3. pdf

21. Gruber, P.G., Nachbagauer, K., Vetyukov, Y., Gerstmayr, J.: A novel director-based Bernoulli-Euler beam finite element in absolute nodal coordinate formulation free of geometric singularities. Mech. Sci. 4, 279-289 (2013). https://doi.org/10. 5194/ms-4-279-2013

22. Meier, C., Grill, M.J., Wall, W.A., Popp, A.: Geometrically exact beam elements and smooth contact schemes for the modeling of fiber-based materials and structures. Int. J. Solids Struct. 154, 124-146 (2018)

23. Vetyukov, Y.: Finite element modeling of Kirchhoff-Love shells as smooth material surfaces. ZAMM 94(1-2), 150-163 (2014b). https://doi.org/10.1002/zamm.201200179

24. Eliseev, V.V.: The non-linear dynamics of elastic rods. J. Appl. Math. Mech. (PMM) 52(4), $493-498$ (1988)

25. Antman, S.S.: The theory of rods. In: Flügge, S., Truesdell, C. (eds.) Handbuch der Physik, vol. VIa/2, pp. 641-703. Springer, Berlin (1972)

26. Singh, P., Goss, V.G.A.: Critical points of the clamped-pinned elastica. Acta Mech. 229, 4753-4770 (2018) 
27. Vetyukov, Yu.: The theory of thin-walled rods of open profile as a result of asymptotic splitting in the problem of deformation of a noncircular cylindrical shell. J. Elast. 98(2), 141-158 (2010)

Publisher's Note Springer Nature remains neutral with regard to jurisdictional claims in published maps and institutional affiliations. 